\title{
Automatic measurement and characterization of surface crack defect in steel based on morphological features
}

\author{
Xincheng $\mathrm{Li}^{1, \mathrm{a}}$, Yongchun Tang ${ }^{1, \mathrm{~b}}$, Weixing $\mathrm{Zhu}^{2, \mathrm{c}}$, Xiaoli Wang ${ }^{1}$, Chengwei \\ Zhang $^{1}$, Xudong Liu ${ }^{1}$, Liping Zhu ${ }^{1}$ \\ ${ }^{1}$ School of Mechanical Engineering, Jiangsu University, Zhenjiang 212013, China; \\ ${ }^{2}$ School of Electrical Engineering, Jiangsu University, Zhenjiang 212013, China. \\ alixincheng@ujs.edu.cn, blarrytyc@163.com, 'chuweixing@126.com.
}

\begin{abstract}
Keywords: morphological features; crack defect; measurement; characterization; linear morphological factor.
\end{abstract}

\begin{abstract}
In order to evaluate the effect of size and shape of crack defect on steel surface quality and mechanical properties, accurate measurement and characterization should be conducted. Aimed at the high measuring difficulty and low accuracy in the existing manual mode, a new series of characteristic parameters was proposed according to the morphological features of crack defect in steel. Through the quantitative analysis of parameters including linear morphological factor, defect equivalent and defect rate, the automatic measurement and numerical characterization of crack defect were realized. The results show that the above method can completely solve the high measuring difficulty of complex crack defect distributed with blocky inclusion and crack, which provides a reliable basis for the quantitative analysis of steel surface quality.
\end{abstract}

\section{Introduction}

With the rapid development of iron and steel industry, the effect of hot-rolled steel was more prominent as a new product. However, because of the metallurgical production process and process element long, quality problems always appeared such as surface crack, surface crack blocky inclusions and interwoven type defects [1,2], which had a serious impact on the steel quality and performance, the quality problem could not be avoided by many mills and was considered to be the common problem and difficult to eliminate.

Research on surface defect of hot-rolled steel has gone through complicated and tortuous development process, which was conducted by many factors, such as the metallurgical and evolution process, the microscopic characteristics were very complex, which was puzzled by the capture of the defect and its site record, especially the formation reason of the defect and the essential differences in morphological characteristics, how to measure and characterize it accurately, and then carry on the effective control, there is no systematic research report so far $[3,4]$.

For the measurement and characterization of surface crack defect in hot-rolled slab, the production site still remained in the manual mode at present, which was characterized by measuring the length and width of the defect area. However, for the more complex crack defect, which combines the massive inclusions and linear cracks, the manual mode is difficult to get the result, which only used a rough statistical defect area as defect characterization parameters. Obviously, the result is too subjective and rough, and will inevitably bring about problems like low accuracy, efficiency and the waste of human resources.

In this paper, a new automatic measurement and characterization method is proposed on the basis of morphological features, in order to realize the measurement of surface crack defect and provide a reliable basis to the quantitative analysis of steel surface quality.

\section{Automatic measurement and characterization theory of surface crack defect}

Firstly, according to the crack defect in target image, a new classification method of minimum defect branch based on the morphological characteristics was established to characterize the size 
and breakage degree of surface defect. Segment the corner points (morphological characteristics of surface defect), get all the corner points (a total of $n$ including the first and last point) and the minimal segments constituted by these points (n-1 segments). Secondly, carry out the region labeling of each minimal segment in target image, the target image region labeling would be most satisfactory if each minimal segment shape uses the most close to the minimum circumscribed box shape (minimum circumscribed rectangle or circle). Therefore, this paper puts forward the linear morphological factor determination method, which is used for the form classification and region labeling of all the minimum section, then use the corresponding minimum circumscribed rectangle and minimum circumscribed circle to calibrate all the minimum sections on the basis of linear morphological factor threshold. Lastly, after completing the minimum segment classification and region labeling work, calculate the summation of all minimum section area by the establishment and quantitative analysis of morphological characteristics, such as defect equivalent, defect rate and so on, so as to complete the automatic measurement and numerical characterization of surface crack defect. Shown in Fig.1 (a), this paper selected a crack defect image for further research, which was resulted from sulfide inclusion, combines with massive inclusion and linear cracks.
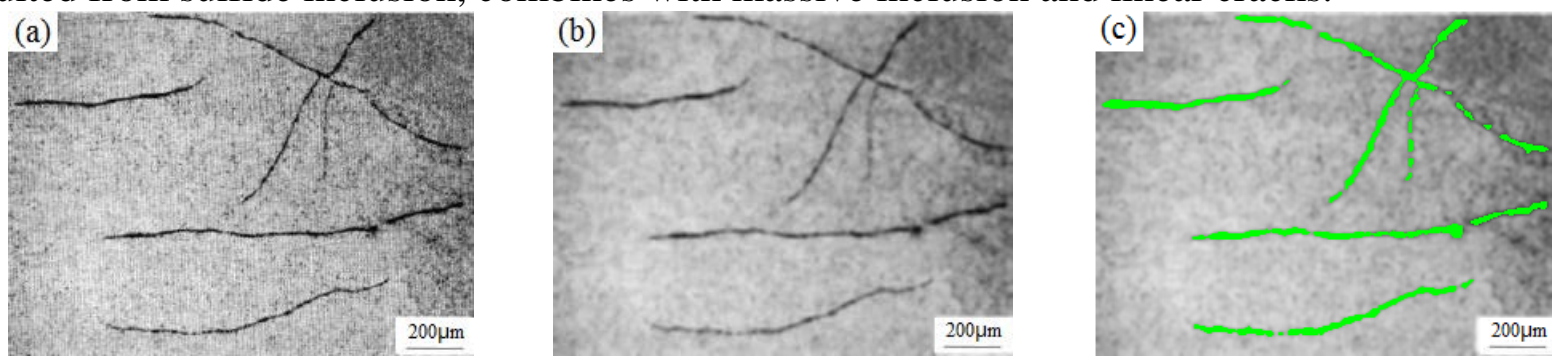

Fig.1 Pre-treated effect of surface crack defect image

(a) Original image; (b) low-pass filtered; (c) self-adaptive threshold segmented

\section{Image pretreatment, classification and region labeling of minimum defect branch}

Image pretreatment and binary segmentation. The effect of image pretreatment is to highlight the useful information and weaken or remove the secondary information in image, so as to improve the image quality. In this paper, a linear average low-pass filtered algorithm is used to pre-treat the original image in Fig.1 (a), filter out the noise contained in original image, which will enhance the image smoothing effect, the pretreated effect is shown in Fig.1 (b).

The effect of binary segment is to separate the target from background, so as to better identify the target in image. An adaptive threshold method based on region partition is proposed in this study [5], which is used to get the ideal gray threshold. In this method, the image is divided into sub blocks, and the maximum variance of each sub block is calculated respectively. In this way, the optimal gray threshold can be obtained automatically, and the segmentation result will be the best. The adaptive threshold segmentation effect of Fig.1 (b) is shown in Fig.1 (c).

Classification and region labeling of minimum defect branch. To recognize the defect kind accurately, morphology classification of all minimum segments should be conducted, find the minimum circumscribed boxes which are most close to minimum segments, and then carry out the region labeling accurately. For this purpose, a linear morphological factor method was proposed to classify the defect form, the formula for calculating the linear form factor $\mathrm{C}$ is shown in Formula 1.

$$
C=\frac{L}{B}
$$

In Formula 1, $\mathrm{L}$ means the length of minimum circumscribed rectangle of the minimum segments, and B means the width of minimum circumscribed rectangle of the minimum segments.

According to the calculated threshold of linear morphological factor $\mathrm{C}$, all minimal segments can be effectively classified. A large number of experimental results show that, the best classification threshold and the corresponding minimal section forms are as follows: (1)when linear morphological factor $\mathrm{C} \geq 4$, linear defects; (2)when $C<4$, massive defects. The former should be region-labeled with the corresponding minimum circumscribed rectangle, while the rest should be 
region-labeled with the minimum circumscribed circle. The minimum segment classification and region labeling effect of Fig.1 (c) are shown in Fig.2.

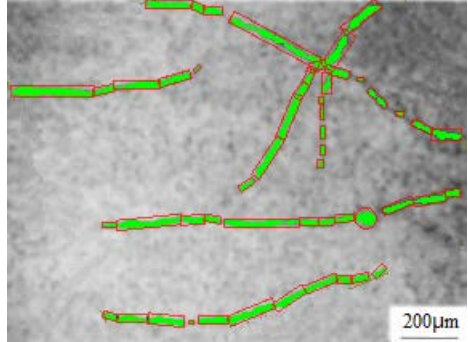

Fig.2 Classified and region demarcated effect of minimum defect branch

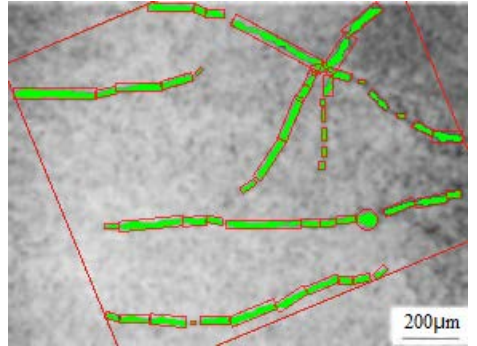

Fig.3 Schematic diagram of defect rate

\section{Establishment and extraction of defect characterization parameters}

While characterizing and measuring the steel crack defect, common geometric quantity should be adopted to calculate conveniently as much as possible, such as length, width, linear morphological factor, area and so on. However, to characterize the size and damage extent of surface defect more accurately, the above common geometric quantities cannot achieve the desired effect obviously. Therefore, this study explored a new series of characterization parameters of surface crack defect on the basis of massive analysis of defect characteristics, the concrete expression as follows:

(1)Defect equivalent $\left(S_{D E}\right)$

$$
S_{D E}=\sum_{i=1}^{n} L_{i} B_{i}+\sum_{j=1}^{m} \frac{1}{4} \pi d_{j}^{2}
$$

In Formula 2, $\mathrm{n}$ means the total of minimum circumscribed rectangle, $\mathrm{m}$ means the total of minimum circumscribed circle, $d_{j}$ means the diameter of minimum circumscribed circle; $S_{D E}$ means the area summation of minimum circumscribed boxes of all minimal sections in test image, namely meaning the total area of defect, the dimension was consistent with the original image.

(2) The defect rate $(\rho)$, the calculation formula and diagram are shown in Fig.3 and Formula 3.

$$
\rho=\frac{S_{D E}}{A_{0}}
$$

In Formula $3, A_{0}$ means the area of minimum circumscribed rectangle covering the whole measured defect (shown in Fig.3), $\rho$ particularly means the ratio of $S_{D E}$ and $A_{0}$ in test image.

\section{Measurement and characterization result of surface cracking type defects}

Now apply the new measurement and characterization method of this paper to the original image (Fig.1), and the measurement and characterization results are shown in Table 1 that, the measuring accuracy of this new method reaches up to $\pm 0.001 \mu \mathrm{m}$, which is the highest measurement accuracy in current analysis of surface quality. In addition, the whole measurement and characterization process are run in a computer with standard configuration. As the measurement and characterization process needs no manual intervention, the efficiency raises up to hundreds of times when comparing with manual mode, and the whole defect analysis in one field of view only needs 26 seconds. Thus in industrial production, the measurement and characterization requirement of massive quantity and complex form can be fully satisfied both in precision and timeliness.

Table1 Measurement and characterization result of surface crack defect

\begin{tabular}{ccccc}
\hline$L_{\max } / \mu \mathrm{m}$ & $B_{\max } / \mu \mathrm{m}$ & $d / \mu \mathrm{m}$ & $S_{D E} / \mu \mathrm{m}^{2}$ & $\rho / \%$ \\
\hline $3.657 \times 10^{2}$ & 52.941 & 89.283 & $1.774 \times 10^{5}$ & 9.660
\end{tabular}

$L_{\max }, B_{\max } d$ in Table 1 respectively means the maximum chord length, the maximum width of minimal segment, and the diameter of minimum circumscribed circle. 


\section{Conclusion}

(1) Accurate classification and region labeling could be realized by the classification method of minimum defect branch, which ensured the following extraction and accurate calculation of defect characteristic parameters.

(2) On the basis of the classification method above, a new series of characterization parameters and special formulas, including defect equivalent and defect rate were proposed to solve the high measuring difficulty of complex crack defect distributed with blocky inclusion and crack, which realized the automatic measurement and numerical characterization of crack defect.

(3) Application result showed that, the automatic method referred in this paper can efficiently realize the measurement of surface crack defect (the precision reached up to $\pm 0.001 \mu \mathrm{m}$ ), which provided a reliable basis to the quantitative analysis of steel surface quality. This method has a great universality, and could be popularized to apply in the field of material, road, building, and so on.

\section{Acknowledgement}

In this paper, the research was sponsored by A Project Funded by the Priority Academic Program Development of Jiangsu Higher Education Institutions and the Graduate innovative project of Jiangsu Province (CXLX11_0561).

\section{References}

[1] Peng Kai, Liu Yazheng, Xie Bin, et al. Analysis of scar defect of hot rolled strip, J. Iron and Steel, 2007, 42(3): 44-46.

[2] Hiroshi U, Kenichiro H, Ryo M, et al. Formation mechanism of surface scale defects in hot rolling process, J. CIRP Annals - Manufacturing Technology, 2014, 63, 261-264.

[3] Zhang Guoyi, Hu Zheng, Xu Ting. Classification method for defect image based on feature extraction, J. Journal of Beijing University of Technology, 2010, 36(4): 450-457.

[4] Sun Bin, Liu Zhenyu, Wang Guodong. Classification and their formation mechanism of typical rolled-in scales on hot rolled plates, J. Journal of Northeastern University, 2010, 31(10): 1417-1420.

[5] Li Xincheng, Ma Zhengjian, Zhu Weixing, et al. Measurement and classification of full morphology grain in ultra-fine grain steel based on morphological features, J. Transactions of Materials and Heat Treatment, 2015, 35(7): 218-221. 